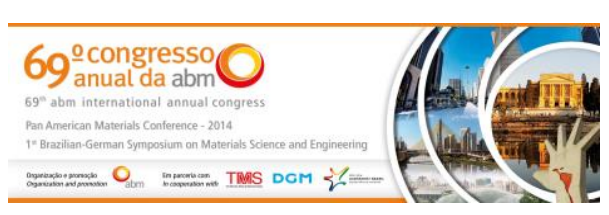

Tema: Materiais Cerâmicos, Compósitos e Poliméricos

\title{
FORMULAÇÃO NUMÉRICA EM ELEMENTOS FINITOS DO AMORTECIMENTO EM MATERIAIS COMPÓSITOS USANDO A TEORIA DA DEFORMAÇÃO CISALHANTE DE PRIMEIRA ORDEM (FSDT)*
}

\section{Resumo}

Adriana Amaro Diacenco ${ }^{1}$ Débora Junqueira Fonseca² Camila Aparecida Diniz ${ }^{3}$

O presente trabalho propõe-se a analisar a influência do amortecimento estrutural inerente de tais materiais submetidos a perturbações estáticas e dinâmicas as quais afetam as características de resposta. A importância deste trabalho insere-se no contexto do controle de vibrações de sistemas de engenharia. Para tanto, é empregada uma técnica de modelagem baseada em elementos finitos. A análise numérica é desenvolvida utilizando a Teoria da Deformação Cisalhante, a qual é implementada em um elemento Serendipity retangular contendo oito nós. $O$ amortecimento é incluído empregando o modelo histerético. As respostas dinâmicas são representadas por funções de resposta em freqüência e autovalores complexos. Simulações numéricas são realizadas visando quantificar os níveis de amortecimento obtidos no tocante às vibrações transversais de placas retangulares e verificar a possibilidade de se aumentar tal amortecimento.

Palavras-chave: Amortecimento inerente; Material compósito; Elementos finitos; FSDT.

\section{FINITE ELEMENT NUMERICAL FORMULATION IN DAMPING IN COMPOSITE MATERIALS USING THE THEORY OF DEFORMATION SHEAR FIRST ORDER}

\section{Abstract} (FSDT)

This paper proposes to examine the influence of the inherent structural damping of such materials under static and dynamic disturbances which affect the response characteristics. The importance of this work is within the context of vibration control systems engineering. For this, we use a modeling technique based on finite elements. Numerical analysis is performed using the Shear Deformation Theory, which is implemented in a rectangular Serendipity element containing eight nodes. Damping is included employing the hysteretic model. The dynamic responses are represented by functions of frequency response and complex eigenvalues. Numerical simulations are performed to quantify the levels of damping obtained with respect to the transverse vibrations of rectangular plates and check the possibility to increase this damping.

Keywords: Inherent damping; Composite material; Finite elements; FSDT.

1 Licenciada em Física Licenciatura, Mestre em Ciência e Engenharia de Materiais, Professora Pesquisadora, Núcleo de Pesquisa Institucional, FEPI, Centro Universitário de Itajubá, Itajubá, MG, Brasil.

2 Graduanda em Engenharia Mecânica, Bolsista, FAPEMIG, FEPI, Itajubá, MG, Brasil.

3 Graduanda em Engenharia de Produção, Bolsista, FAPEMIG, FEPI, Itajubá, MG, Brasil.

\footnotetext{
* Contribuição técnica ao $69^{\circ}$ Congresso Anual da ABM - Internacional e ao 14ํㅡㄹ ENEMET - Encontro Nacional de Estudantes de Engenharia Metalúrgica, de Materiais e de Minas,21 a 25 de julho de 2014, São Paulo, SP, Brasil.
} 


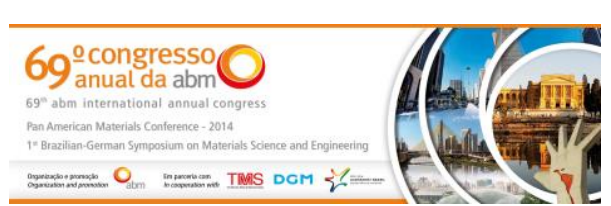

\section{INTRODUÇÃO}

Materiais compósitos formados por matrizes poliméricas apresentam a vantagem de atenuar maiores níveis de vibração, pois os materiais poliméricos possuem natureza viscoelástica que pode ser explicado devido ao fato de polímeros apresentarem um significativo componente viscoso, responsável por dissipar parte da energia cinética, convertendo-a em calor através do movimento interno de suas macromoléculas [1].

Atualmente, os materiais compósitos têm sido amplamente utilizados em diversos áreas da engenharia, destacando-se no setor aeroespacial. A empresa Boeing lançou em 2007 o modelo 787, o Dreamliner, cuja estrutura primária, incluindo fuselagem e asas, é $50 \%$ feita em material compósito [2].

O estudo e análise do comportamento de materiais compósitos tem motivado inúmeros pesquisadores [3-5] e ensaios destes materiais em escala real podem se tornar inviáveis devido ao custo ou tempo e isto tem impulsionado diversos modelos numéricos que possibilitem a representação do comportamento de tais materiais.

De acordo com o exposto acima, o objetivo deste trabalho é descrever a modelagem do comportamento de materiais compósitos e considerar a influência do amortecimento inerente no equacionamento. Para tanto é empregada uma Teoria de Primeira Ordem combinada com um elemento finito de placa plana. Toda a modelagem matemática será avaliada computacionalmente utilizando o ambiente de programação $M A T L A B^{\circledR}$, e os resultados obtidos permitiram avaliar o desempenho do modelo utilizado.

\section{DESCRIÇÃO DO AMORTECIMENTO INERENTE}

Materiais que possuem o comportamento viscoelástico podem ser caracterizados de modo dinâmico através da seguinte expressão:

$$
G(\omega)=G^{\prime}(\omega)+i G^{\prime \prime}(\omega)=G^{\prime}(\omega)[1+i \eta(\omega)]
$$

Onde: o módulo complexo do material. $G^{\prime}(\omega)$ e $G^{\prime \prime}(\omega)$ são, respectivamente, os módulos de armazenamento e de perda do material, e $\eta(\omega)=G^{\prime \prime}(\omega) / G^{\prime}(\omega)$ é o fator de perda do material (capacidade de amortecimento do material viscoelástico).

A Figura 1 apresenta comportamento viscoelástico em função da temperatura [6].

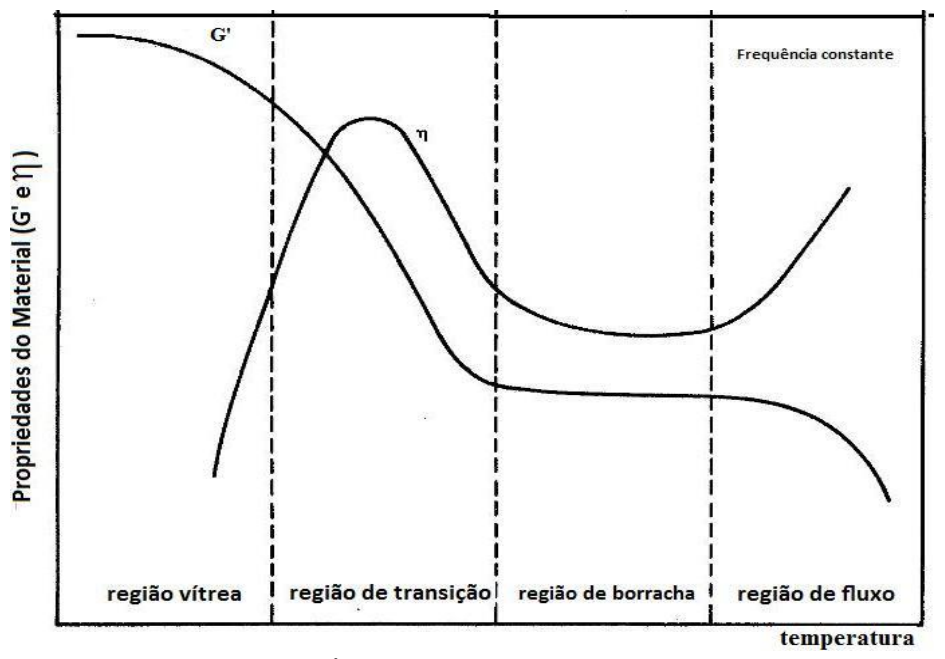

Figura 1. Variação de $G^{\prime}$ e $\eta$ com a temperatura (adaptado de [6]).

\footnotetext{
* Contribuição técnica ao $69^{\circ}$ Congresso Anual da ABM - Internacional e ao 14ํㅡㄹ ENEMET - Encontro Nacional de Estudantes de Engenharia Metalúrgica, de Materiais e de Minas,21 a 25 de julho de 2014, São Paulo, SP, Brasil.
} 
Segundo Diacenco [2], nota-se que a segunda região é caracterizada por um fator de perda máximo, fato que garante um amortecimento eficiente, a terceira região demonstra-se bastante atrativa, visto que o módulo de armazenamento e o fator de perda variam muito pouco com a temperatura, apresentando também resultados satisfatórios com relação à capacidade de amortecimento.

Descrever detalhadamente os equipamentos e os procedimentos utilizados, permitindo que a pesquisa possa ser reproduzida posteriormente por outros pesquisadores. Apresentar a literatura e a descrição dos métodos estatísticos empregados, quando for o caso.

\section{MODELAGEM POR ELEMENTOS FINITOS}

O campo de deslocamentos mecânicos representado pela Teoria da Deformação Cisalhante de Primeira Ordem é expresso por:

$$
\begin{aligned}
& u(x, y, z, t)=u_{0}(x, y, t)+z \psi_{x}(x, y, t) \\
& v(x, y, z, t)=v_{0}(x, y, t)+z \psi_{y}(x, y, t) \\
& w(x, y, z, t)=w_{0}(x, y, t)
\end{aligned}
$$

onde: $\Psi_{\mathrm{x}}$ e $\Psi_{\mathrm{y}}$ são as rotações dos segmentos normais à superfície de referência em torno dos eixos y e $\mathrm{x}$ respectivamente.

As variáveis mecânicas apresentadas na Equação (2) são convertidas para sua formulação em elementos finitos usando funções de forma apropriadas. O elemento considerado, ilustrado na Figura 2, é um elemento retangular de placa que apresenta três nós por aresta, perfazendo um total de 8 nós, sendo conhecido como elemento Serendipity [3].

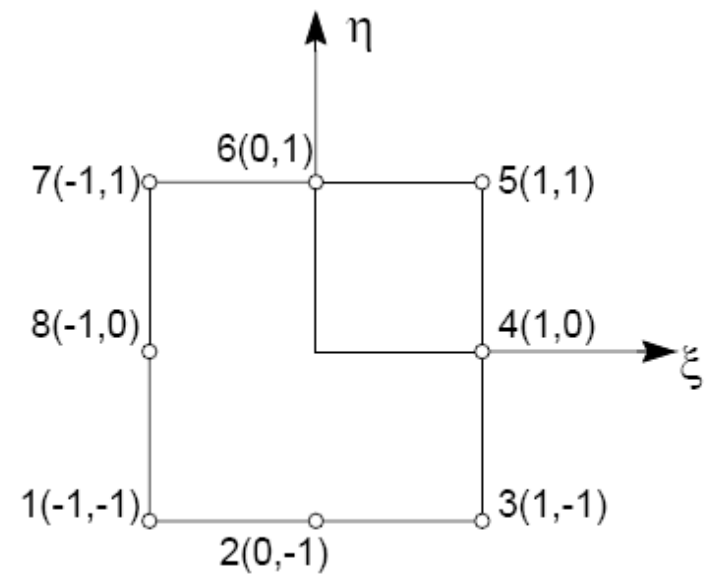

(A)

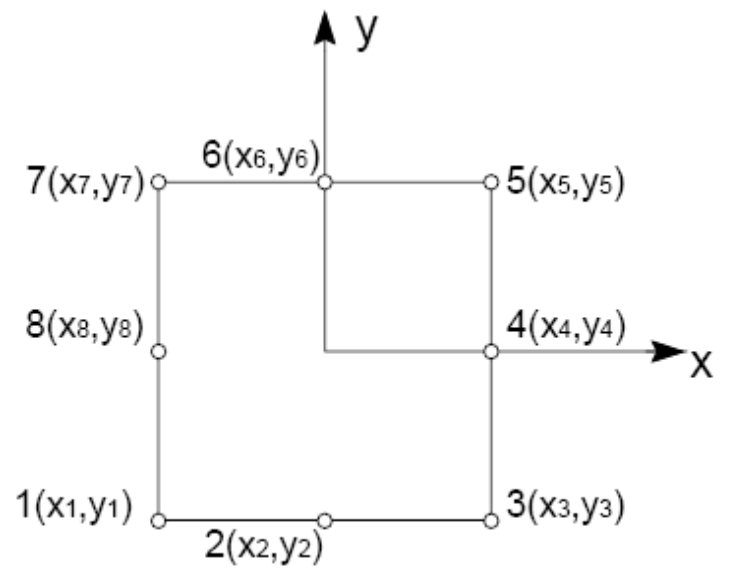

(B)

Figura 2. Forma retangular do elemento empregado na formulação por elementos finitos (a) coordenadas locais (b) coordenadas globais.

As funções de forma, que relaciona os deslocamentos em um ponto qualquer com os deslocamentos nos pontos nodais, da família Serendipity são apresentadas na Equação (3).

\footnotetext{
* Contribuição técnica ao 69ำ Congresso Anual da ABM - Internacional e ao 14ํㅡㄹ ENEMET - Encontro Nacional de Estudantes de Engenharia Metalúrgica, de Materiais e de Minas,21 a 25 de julho de 2014, São Paulo, SP, Brasil.
} 


$$
\begin{aligned}
& N_{1}(\xi, \eta)=-\frac{1}{4}(1-\xi)(1-\eta)(1+\xi+\eta) \\
& N_{2}(\xi, \eta)=\frac{1}{2}(1-\xi)(1+\xi)(1-\eta) \\
& N_{3}(\xi, \eta)=-\frac{1}{4}(1+\xi)(1-\xi)(1-\xi+\eta) \\
& N_{4}(\xi, \eta)=\frac{1}{2}(1+\xi)(1+\eta)(1-\eta) \\
& N_{5}(\xi, \eta)=-\frac{1}{4}(1+\xi)(1+\eta)(1-\xi+\eta) \\
& N_{6}(\xi, \eta)=\frac{1}{2}(1-\xi)(1+\xi)(1+\eta) \\
& N_{7}(\xi, \eta)=-\frac{1}{4}(1-\xi)(1+\eta)(1+\xi-\eta) \\
& N_{8}(\xi, \eta)=\frac{1}{2}(1-\xi)(1+\eta)(1-\eta)
\end{aligned}
$$

A expressão (2) pode ser escrita em termos das funções de forma como:

$$
\{\hat{u}(\xi, \eta, t)\}_{(5 x x)}=\left[N_{u}(\xi, \eta, t)\right]_{5 \times 40}\left\{u_{e}(t)\right\}_{40 \times 1}
$$

Da Teoria da Elasticidade Linear tem-se que as deformações mecânicas são definidas em termos das formas diferenciais dos deslocamentos mecânicos [3] expressos na Equação (3):

$$
\begin{aligned}
& \varepsilon_{x x}=\frac{\partial u}{\partial x}, \varepsilon_{y y}=\frac{\partial v}{\partial y}, \varepsilon_{z z}=\frac{\partial w}{\partial z} \\
& \gamma_{x y}=\frac{\partial u}{\partial y}+\frac{\partial v}{\partial x}, \gamma_{x z}=\frac{\partial u}{\partial z}+\frac{\partial w}{\partial y}, \gamma_{y z}=\frac{\partial v}{\partial z}+\frac{\partial w}{\partial y}
\end{aligned}
$$

Utilizando as relações expressão na Equação 4 e separando os efeitos em efeito de flexão-membrana e cisalhamento obtém-se:

$\varepsilon_{b}(x, y, z, t)=\left[D_{0}+z D_{1}\right] u(x, y, t)=D_{b}(z) u(x, y, t)$

$\varepsilon_{s}(x, y, z, t)=\left[D_{2}\right] u(x, y, t)=D_{s}(z) u(x, y, t)$

As matrizes $D_{i}(i=0, \ldots, 2)$ são formadas por operadores diferenciais.

Podemos expressar as deformações de flexão $\varepsilon$ b e deformações de cisalhamento transversais $\varepsilon_{\mathrm{s}}$ em coordenadas locais, obtendo-se:

$\varepsilon_{b}(\xi, \eta, t)=\left[D_{b}(z)\right]\left[N_{u}(\xi, \eta)\right]\left\{u_{e}(t)\right\}$

$\varepsilon_{s}(\xi, \eta, t)=\left[D_{s}(z)\right]\left[N_{u}(\xi, \eta)\right]\left\{u_{e}(t)\right\}$

As energias, cinética e de deformação, na formulação em elementos finitos são expressas por:

$$
\begin{aligned}
& K_{E}=\frac{1}{2} u^{\prime T}(t) M^{(e)} u^{\prime}(t) \\
& S_{E}=\frac{1}{2} u^{\prime T}(t) K^{(e)} u^{\prime}(t)
\end{aligned}
$$

\footnotetext{
* Contribuição técnica ao 69ำ Congresso Anual da ABM - Internacional e ao 14ํㅡㄹ ENEMET - Encontro Nacional de Estudantes de Engenharia Metalúrgica, de Materiais e de Minas,21 a 25 de julho de 2014, São Paulo, SP, Brasil.
} 
Onde:

$M^{(e)}=\sum_{k=1}^{n} \int_{z=z_{k}}^{z_{k}+1} \int_{\xi=-1}^{\xi=+1} \int_{\eta=-1}^{\eta=+1} \rho_{k} N^{T}(\xi, \eta) A^{T}(z) A(z) N(\xi, \eta) \operatorname{det}(J) d \eta d \xi d z$

onde $\mathrm{M}^{(\mathrm{e})}$ é a matriz de massa elementar, e $\mathrm{K}^{(\mathrm{e})}=\mathrm{K}_{\mathrm{b}}(\mathrm{e})+\mathrm{K}_{\mathrm{s}}(\mathrm{e})$ é a matriz de rigidez elementar, considerando os efeitos de flexão-membrana e de cisalhamento expressados por:

$$
\begin{aligned}
& K_{b}{ }^{(e)}=\sum_{k=1}^{n} \int_{z=z k}^{z k+1} \int_{\xi=-1}^{\xi=+1} \int_{\eta=-1}^{\eta=+1} B_{b}^{T}(\xi, \eta, z) C_{b}{ }^{(k)}\left(\theta_{k}\right) B_{b}(\xi, \eta, z) \operatorname{det}(J) d \eta d \xi d z \\
& K_{s}{ }^{(e)}=\sum_{k=1}^{n} \int_{z=z k}^{z k+1} \int_{\xi=-1}^{\xi=+1} \int_{\eta=-1}^{\eta=+1} B_{s}^{T}(\xi, \eta, z) C_{s}{ }^{(k)}\left(\theta_{k}\right) B_{s}(\xi, \eta, z) \operatorname{det}(J) d \eta d \xi d z
\end{aligned}
$$

As matrizes $\mathrm{C}_{b}{ }^{\mathrm{k}}(\theta)$ e $\mathrm{C}_{\mathrm{s}}{ }^{\mathrm{k}}(\theta)$ são ortotrópicas e estão em função do ângulo de orientação das fibras, ou seja, o material compósito está em função das coordenadas locais, assim é conveniente utilizar uma matriz de transformação, para promover a transformação do sistema de coordenadas locais do material para coordenadas globais.

A partir do cálculo das matrizes elementares podemos escrever a equação global do movimento no domínio do tempo da seguinte forma:

$M \ddot{q}(t)+K q(t)=f(t)$

onde $M=\bigcup_{e=1}^{\text {nelem }} M^{(e)}$ e $K=\bigcup_{e=1}^{\text {nelem }} K^{(e)}$ são as matrizes de rigidez global e de massa. O símbolo $U$ indica a matriz de montagem, $q(\mathrm{t})$ é o vetor de deslocamento global e $f(t)$ é o vetor de cargas generalizadas.

A Equação 11 pode ser expressa pela conforme a equação a seguir e assim tem-se a matriz de rigidez dinâmica complexa no domínio da frequência:

$$
Z(\omega)=K_{e}+G(\omega) \bar{K}_{v}-\omega^{2} M
$$

É assumido que o material compósito apresenta amortecimento inerente, representado pelos módulos complexos e independentes da frequência e temperatura da seguinte forma:

$$
E_{m n}=\bar{E}_{m n}\left(1+i \eta_{m n}\right), \quad E_{m n}=\bar{G}_{m n}\left(1+i \eta_{m n}\right)
$$

\section{RESULTADOS NUMÉRICOS}

\subsection{Aplicação 1}

Os resultados serão expressos por Funções de Resposta em Frequência que representaram as amplitudes de vibração (eixo y) e as frequências (eixo x).

Considerou-se uma placa plana composta laminada, de dimensões denominadas de $L_{x}=L_{y}=0.20 \mathrm{~m}$, composta por cinco camadas unidirecionais de mesma espessura e igual a $h / 5$, onde $h=L_{x} / 140$. A placa foi discretizada em 64 elementos finitos com as seguintes condições de contorno: $u_{0}=w_{0}=\psi_{z}=\varsigma_{x}=\varsigma_{z}=0$ em y $=0$ e y $=a$, e

* Contribuição técnica ao $69^{\circ}$ Congresso Anual da ABM - Internacional e ao 14ํㅡㄹ ENEMET - Encontro Nacional de Estudantes de Engenharia Metalúrgica, de Materiais e de Minas,21 a 25 de julho de 2014, São Paulo, SP, Brasil. 
$\mathrm{u}_{0}=\mathrm{w}_{0}=0$ em $\mathrm{x}=0$ e $\mathrm{x}=\mathrm{b}$ [7]. As orientações das fibras de cada camada são adotadas arbitrariamente como (45\%/0\%/45\%/0\%/45). As propriedades elásticas de cada camada são: $\quad \bar{E}_{1}=172,4 G P a, \quad \bar{E}_{2}=\bar{E}_{3}=6,89 G P a, \quad \bar{G}_{12}=\bar{G}_{13}=3,45 G P a$, $\bar{G}_{23}=1,38 G P a, v_{12}=v_{13}=0,25, v_{23}=0,30, \rho=1566 \mathrm{~kg} / \mathrm{m}^{3}$ é a densidade do material.

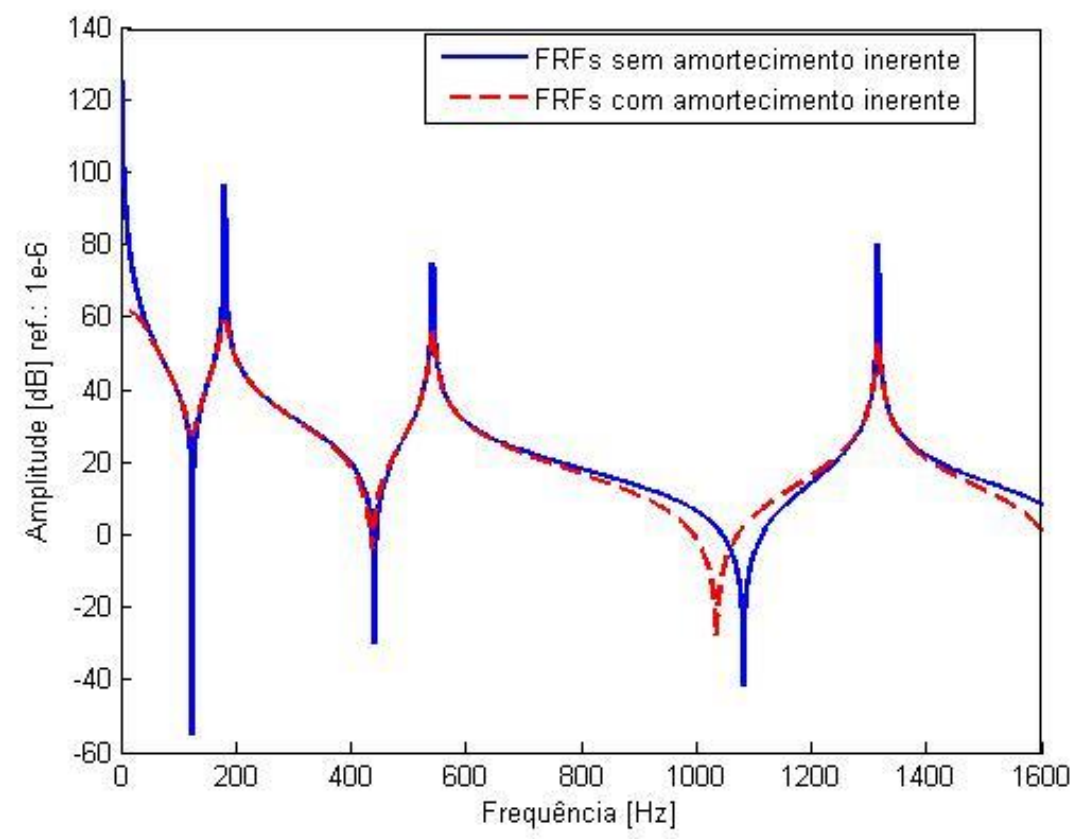

Figura 3. FRFs para duas situações distintas.

Foram consideradas duas situações distintas onde foi possível obter as funções de resposta em frequência desconsiderando o amortecimento inerente e considerando 0 amortecimento inerente, onde se adotou o fator de perda como sendo $\eta_{m n}=0.0005$, visto que é o fator de perda que representa a capacidade de amortecimento do material. Neste exemplo, nota-se que mesmo o valor do fator de perda sendo pequena sua influência no modelo é indispensável, logo todos os materiais que possuem em sua constituição materiais que apresentam algum tipo de comportamento viscoelástico devem ser modelados com a consideração do fator de perda no modelo.

\subsection{Aplicação 2}

Para ilustrar o desempenho do modelo nesta sessão considerou-se a presença do amortecimento inerente em duas camadas do material compósito. As considerações geométricas e mecânicas são as mesmas da aplicação anterior.

\footnotetext{
* Contribuição técnica ao 69ำ Congresso Anual da ABM - Internacional e ao 14ํㅡㄹ ENEMET - Encontro Nacional de Estudantes de Engenharia Metalúrgica, de Materiais e de Minas,21 a 25 de julho de 2014, São Paulo, SP, Brasil.
} 

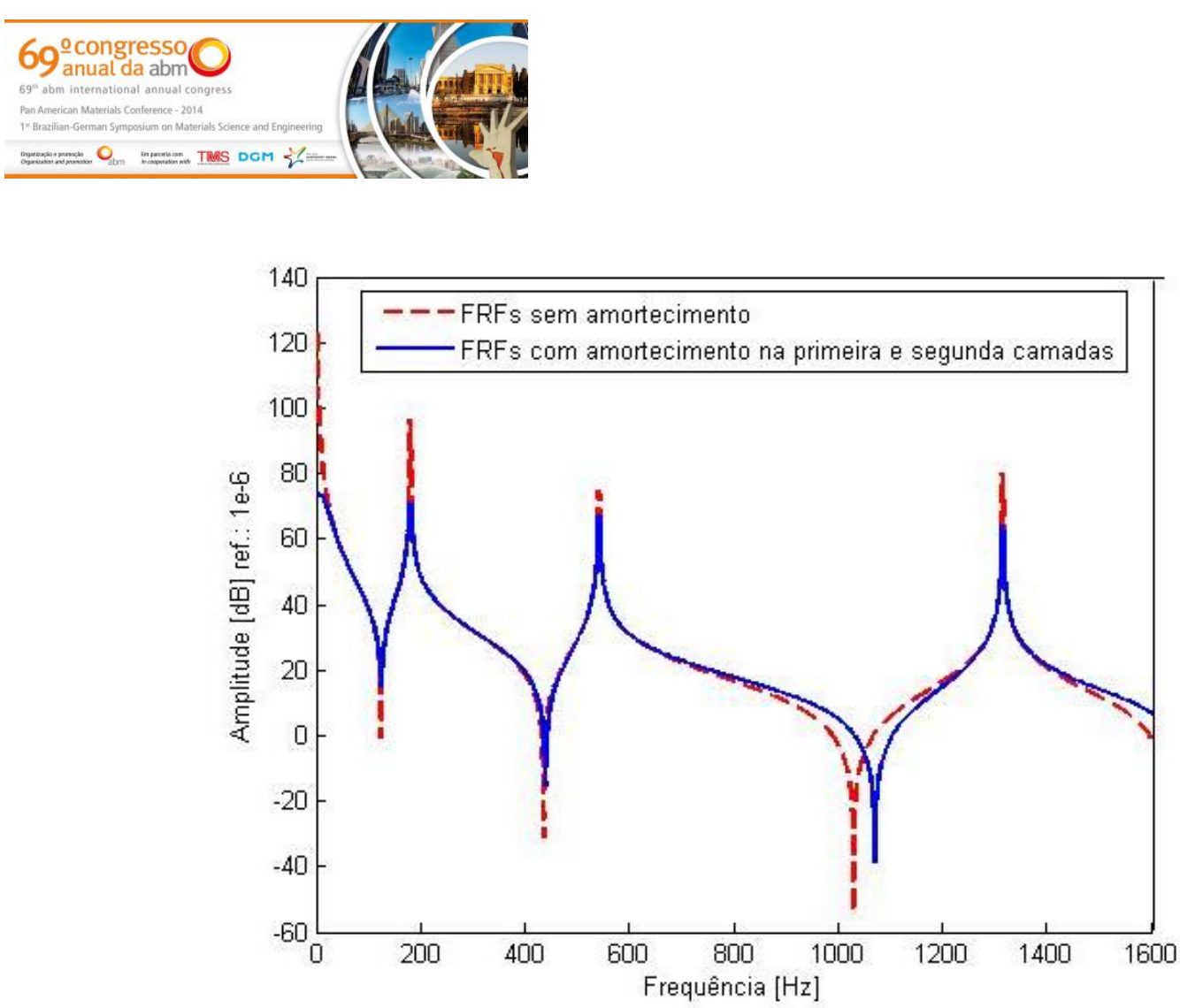

Figura 4. FRFs para duas situações distintas.

Observa-se que a consideração do amortecimento inerente em duas camadas atenua-se os níveis de vibração, mas a consideração em todas as camadas torna-se o modelo mais efetivo.

\section{CONCLUSÃo}

É possível concluir que o modelo implementado consegue descrever a influência do amortecimento inerente e que sua importância destaca-se para a caracterização dinâmica do processo, visto que sua não consideração pode comprometer os resultados do modelo, pois a obter amplitudes de vibração mais elevadas.

\section{Agradecimentos}

Os autores agradecem a Fundação de Amparo a Pesquisa de Minas Gerais (FAPEMIG) pelo apoio ao projeto em desenvolvimento e as bolsas de Iniciação Científica conferida pelas alunas dos cursos de Engenharia da FEPI. E ao Núcleo de Pesquisa Institucional da FEPI - NUPI.

\section{REFERÊNCIAS}

1 Matozo LT. Análise de materiais de fundo de alto amortecimento e de seus efeitos em pastilhas de freios. Porto Alegre: Universidade Federal do Rio Grande do Sul; 2006.

2 Diacenco AA. Modelagem por elementos finitos de materiais compósitos incorporando material viscoelástico para o controle passivo de vibrações e ruído [M.Sc.Dissertation]. Itajubá: UNIFEI; 2010.

3 Reddy JN. Mechanics of Laminated Composite Plates: Theory and Analysis. $2^{\underline{a}}$ ed. Florida: CRC Press; 1997.

4 Lima AMG, Faria AW, Rade DA. Sensitivity analysis of frequency response functions of composite sandwich plates containing viscoelastic layers. Composite Structures. 2010;92:364-376.

* Contribuição técnica ao 69 Congresso Anual da ABM - Internacional e ao 14ํㅡㄹ ENEMET - Encontro Nacional de Estudantes de Engenharia Metalúrgica, de Materiais e de Minas,21 a 25 de julho de 2014, São Paulo, SP, Brasil. 
5 Rade DA, Steffen Jr. V. Absorbers Vibration. In: Braun SG, Ewins DJ, Rao SS (org.). Encyclopedia of Vibration. London: Academic Press; 2001. v.01, p.9-26.

6 Nashif AD, Jones DIG, Henderson JP. Vibration Damping. New York: John Wiley \& Sons; 1985.

7 Correia VMF, Gomes MAA, Suleman A, Soares CMM, Soares CAM. Modelling and Design of Adaptive Composite Structures. Comput. Methods Appl. Mech. Eng. 2000;185:325-346. 